\begin{tabular}{|l|l|}
\hline \multicolumn{2}{|c|}{ Access this article online } \\
\hline
\end{tabular}

\title{
Ab interno goniotomy with a Kahook Dual Blade in 6-month follow-up
}

\author{
Anna Majer ${ }^{1,2}$, Magdalena Kaszuba-Modrzejewska ${ }^{1,2}$, Marzena Petrus ${ }^{1}$, Bartłomiej Kałużnyy, \\ 'Division of Ophthalmology and Optometry, Department of Ophthalmology, Collegium Medicum in Bydgoszcz, Nicolaus Copernicus University \\ in Torun, Poland \\ 2Oftalmika Eye Hospital in Bydgoszcz, Poland
}

\begin{abstract}
Aim of the study: Evaluation of ab interno goniotomy using a Kahook Dual Blade in combination with phacoemulsification in patients with primary open angle glaucoma during a 6-month follow-up.

Material and methods: 75 patients with mild and moderately advanced primary open angle glaucoma, who had phacoemulsification combined with ab interno goniotomy using a Kahook Dual Blade (New World Medical, USA), were included in this analysis. The average age was $68.4 \pm 8.9$ years. Mean pre- and postoperative intraocular pressure, number of antiglaucoma medications, complications of the surgery and need for another procedure were analysed in the 6-month follow-up.

Results: Before the surgery, mean intraocular pressure was $21.9 \pm 3.9$ $\mathrm{mmHg}$ and the number of antiglaucoma medications was $2.2 \pm 1.2$. A reduction in intraocular pressure of $17.53 \%$ and a decrease in
\end{abstract}

number of antiglaucoma medications of $60 \%(p<0.001)$ were observed in the 6-month follow-up. There was slight intra-operative bleeding into the anterior chamber from the incision in all cases. On the first days after surgery there were diffuse blood cells or a clot in the anterior chamber in $38 \%$ of eyes. Increase in intraocular pressure above $25 \mathrm{mmHg}$ on the first day after surgery was observed in $30.6 \%$ of eyes. In one eye there was no postoperative intraocular pressure stabilisation and laser cyclodestruction was performed. Conclusions: $A b$ interno goniotomy performed simultaneously with phacoemulsification results in a significant reduction in intraocular pressure and a decrease in the number of anti-glaucoma drugs used. The treatment is characterized by a good safety profile and does not require intensive postoperative management. KEY WORDS: $a b$ interno goniotomy, MIGS, minimally invasive glaucoma surgery, Kahook Dual Blade, glaucoma.

\section{INTRODUCTION}

An ophthalmologist treating a patient with glaucoma has a choice between several therapeutic modalities including pharmacotherapy, laser therapy, and surgery. Treatment with glaucoma eye drops, which often need to be administered more than once a day, requires the patient's strict compliance with the prescribed regimen and a systematic approach. Another aspect to consider is the fact that in a number of cases glaucoma eye drops are associated with local and systemic side effects. Selective laser trabeculoplasty (SLT) has similar efficacy in lowering intraocular pressure (IOP) as single-drug pharmacotherapy [1]. A definite advantage of this treatment modality is the fact that the therapeutic outcome does not depend on patient compliance. It also has a good safety profile. However, the beneficial effect of treatment does not occur in all patients, and if it does, it declines with time [1]. Laser cyclodestruction has a greater efficacy [2], but the risk of complications is higher than in SLT [3]. Conventional glaucoma procedures, such as trabeculectomy and drainage implants, despite being highly effective, are associated with a considerable risk of potential complications $[4,5]$. In addition, patients undergoing such procedures require close postoperative monitoring. Consequently, the so-called micro-invasive glaucoma surgery (MIGS) has been gaining an increased interest in the last few years [6]. MIGS procedures are an ideal therapeutic solution for patients with early or moderate open-angle glaucoma who do not require intensive and aggressive treatment [4]. Surgical procedures included in this group are performed from the inside, via the transparent part of the cornea ( $a b$ interno). They offer a high safety profile, and have a short healing period. Contrary to classic procedures, they do not require intensive patient monitoring in the postoperative period. The conjunctiva is spared, which does not limit the possibility to perform other procedures in the future [7]. The decision to qualify a patient for this type of surgery can be made at an earlier stage of glaucoma damage than in classic surgical glaucoma procedures. Minimally invasive techniques can be divided into modalities improving

\section{CORRESPONDING AUTHOR}

Magdalena Kaszuba-Modrzejewska MD, Division of Ophthalmology and Optometry, Department of Ophthalmology, Collegium Medicum, Nicolaus Copernicus University, 75 Ujejskiego St., 85-094 Bydgoszcz, e-mail: kaszuba.magdalena@interia.pl 
the trabecular, suprachoroidal and subconjunctival outflow, and reducing aqueous humor production. They can be performed as an isolated procedure or in combination with cataract surgery [6]. The assumption is that the efficacy of MIGS procedures should be comparable to conventional treatments or lower but with fewer potential complications.

The aqueous humor is believed to drain out of the eye via two pathways: conventionally, i.e. through the trabecular meshwork into Schlemm's canal, then passing through the aqueous veins and entering the epidural veins; and unconventionally, i.e. via the uveoscleral pathway. The latter pathway drains up to $45 \%$ of the aqueous humor in young people. However, the importance of this pathway decreases with age, especially in individuals with glaucoma [8]. Another age-related process, attributed to changes in the trabecular meshwork, is an increase in outflow resistance, particularly in glaucoma patients $[8,9]$. Consequently, it appears reasonable to perform procedures aimed at reducing resistance at the trabecular level in patients with primary open-angle glaucoma (POAG), as well as pigmentary or pseudoexfoliation glaucoma.

In 2015, the Food and Drug Administration (FDA) approved the single-use surgical instrument called the Kahook Dual Blade (KDB, New World Medical) for use in minimally invasive glaucoma surgery. The KDB operates by reducing the aqueous humor outflow resistance at the trabecular level. The instrument is equipped with a bent, pointed tip with two side blades (Figure 1).

Kahook Dual Blade is designed to allow surgeons to excise a portion of the trabecular meshwork by making a precise incision and continuing it along Schlemm's canal. The tissue to be excised is lifted on an appropriately profiled ramp and removed from its original position, so that it can be removed from the eye after the procedure is completed. Such controlled excision of the trabecular meshwork establishes a direct connection of the anterior chamber with Schlemm's canal and then communication with the aqueous veins [10, 11]. Through this sequence, the procedure increases the aqueous humor outflow via the conventional pathway. Based on the observations to date, the procedure leads to a reduction in IOP at a level comparable to the effect of treatment with one glaucoma medication. In addition, it allows a decrease in the number of topically administered medications [11].

The aim of the present study is to evaluate the therapeutic outcomes obtained by performing ab interno goniotomy us-

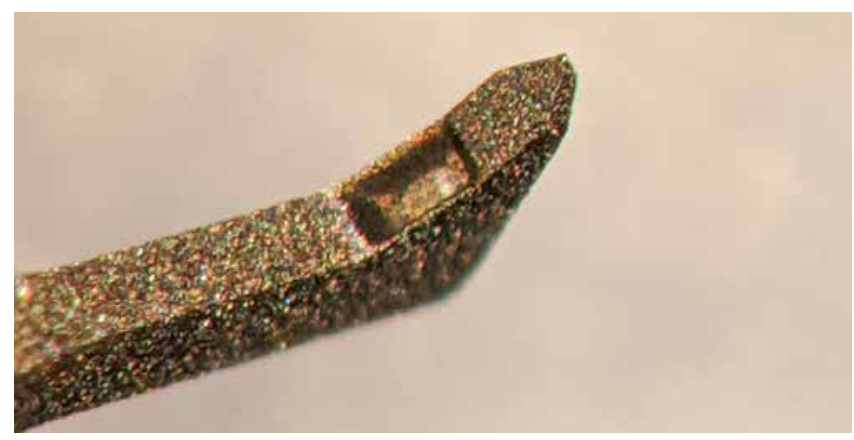

Figure 1. Design of the Kahook Dual Blade ing the KDB in conjunction with phacoemulsification cataract surgery in patients with early and intermediate open-angle glaucoma during a 6-month follow-up. The analysis also included the safety profile of the procedures, and the need for additional treatments.

\section{MATERIAL AND METHODS}

The retrospective study included a total of 75 eyes diagnosed with cataract and POAG which did not require aggressive glaucoma treatment. The mean age of patients was $68.4 \pm 8.9$ years. Women accounted for $56 \%$ of the study group, while $44 \%$ were men. The inclusion criteria were: age over 18 years, early and intermediate POAG coexisting with age-related cataract, and insufficiently effective and/or poorly tolerated pharmacotherapy. The diagnosis of glaucoma was confirmed by detecting characteristic abnormalities in the visual field and RNFL deviations. The study excluded patients with glaucoma types other than POAG, including, but not limited to, patients with pigmentary glaucoma and pseudoexfoliation syndrome, advanced changes in the visual field [MD (absolute value) $>-12 \mathrm{~dB}$, number of points for which $p<5 \%$ is greater than 37 , number of points for which $p<1 \%$ is greater than 20 , absolute defect within the central $5^{\circ}$ of the visual field and presence of points with a sensitivity of $<15 \mathrm{~dB}$ within the central $5^{\circ}$ in both halves of the visual field], patients after laser trabeculoplasty and other surgical glaucoma procedures, failing to report for postoperative follow-up, and patients with advanced systemic conditions.

All patients underwent cataract phacoemulsification with intracapsular implantation of intraocular lens in conjunction with $a b$ interno goniotomy using the Kahook Dual Blade (New World Medical, USA). The indication to extend cataract phacoemulsification was the need to lower IOP or reduce the number of glaucoma medications because of drug intolerance. The procedures were performed by the same surgeon. The patients had follow-up evaluations on the first day after the procedure, after 5-7 days, and then after 1, 3 and 6 months. Intraocular pressure was measured with a Goldman applanation tonometer.

The procedures were conducted under drip anesthesia combined with intrachamber administration of $1 \%$ lignocaine. Goniotomy was performed following the insertion of the intraocular lens into the lens capsule. The opening of Schlemm's canal with a disposable KDB was performed at $100-120^{\circ}$. A direct gonioscope (Volk Surgical ACS Gonio Lens) was used to visualize the filtration angle. In some patients, it was necessary to inject Miostat into the anterior chamber in order to constrict the pupil and pull the iris out of the filtration angle. The anterior chamber was filled with a viscoelastic (2\% methylcellulose). The operating microscope was tilted approximately $45^{\circ}$, and the patient's head was rotated approximately $40^{\circ}$ away from the treated eye. The Kahook blade was inserted via a side port with a width of 1.4-1.6 mm (Figure 2).

The incision of the filtration angle along Schlemm's canal was made at $100-120^{\circ}$, in two steps. The first incision was performed in the inferior quadrant from bottom to top. Next, the 
blade was rotated $180^{\circ}$. The second incision was made in the upper quadrant, some distance from the end of the first incision, and was continued down until the two incisions met, and an excised trabecular meshwork strip was obtained. In this manner, the posterior wall of Schlemm's canal was exposed. The excised portion of the trabecular meshwork was removed from the anterior chamber. After the bleeding from the incision site had stopped, the viscoelastic was rinsed out from the anterior chamber. Figure 3 shows the gonioscopic appearance of the region of the filtration angle after the surgery.

The patient was monitored during the first postoperative day. Until the follow-up, the patient did not use any medications in the operated eye. In patients with good IOP control before the procedure and normal IOP in the follow-up examination, a decision was made to reduce the number of glaucoma medications used. In patients with elevated IOP on the first day, it was lowered, as required; while the number of drugs was left unchanged. Other postoperative recommendations were the same as in cataract surgery, including topical antibiotics, steroids, and non-steroidal anti-inflammatory drugs. At subsequent follow-ups, based on IOP values, the need for further modification of pharmacological glaucoma therapy was determined.

The study endpoints were to achieve an IOP value $\leq 18 \mathrm{mmHg}$ or lower the IOP value by at least $20 \%$ and reduce the number of glaucoma medications used by patients by at least one. Intraocular pressure, the number of glaucoma medications used postoperatively, and the presence of potential complications associated with the procedure were evaluated. As the study had a retrospective observational design, no preoperative withdrawal of glaucoma medications was scheduled as the washout phase. Given the design of the study, no control group was planned, either. The degree of IOP reduction and the number of medications taken by patients after combination surgery were compared with the baseline values and examined against literature data on IOP reduction after cataract phacoemulsification alone.

The compliance of the distribution of values with normal distribution was verified with the Shapiro-Wilk normality test, and descriptive statistics were presented as arithmetic means and standard deviations $( \pm S D)$. The significance of differences between the groups was assessed using Student's $t$-test (for variables with a normal distribution) or MannWhitney's $U$ test (for non-parametric variables). The value of $0.05(p \leq 0.05)$ was adopted as the probability threshold.

\section{RESULTS}

\section{Intraocular pressure changes}

The mean baseline IOP was $21.9 \pm 3.9 \mathrm{mmHg}$. A statistically significant reduction in IOP was observed as early as on the first postoperative day. The effect persisted at subsequent follow-ups. All differences are highly significant statistically in relation to the baseline examination $(p<0.001)$. Table I lists the changes in mean IOP during the follow-up of 6 months. Mean IOP was reduced by $17.53 \%$ from the baseline. The predefined IOP reduction by $20 \%$ was achieved

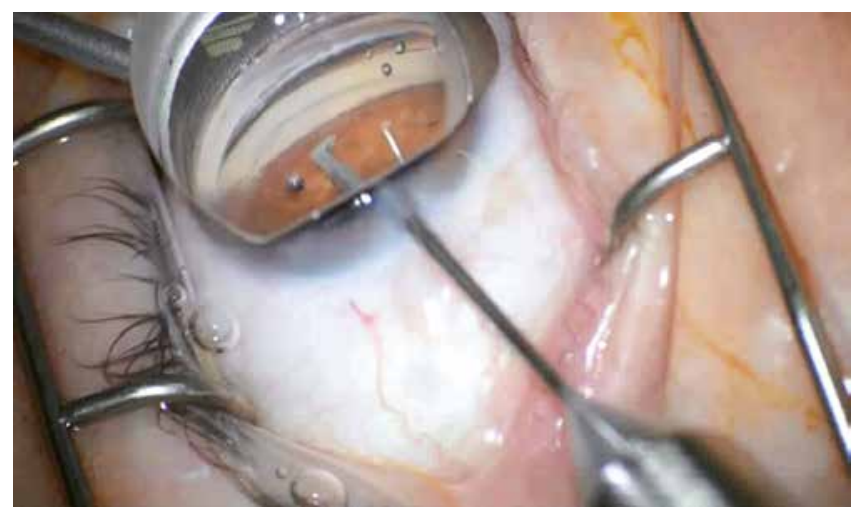

Figure 2. Intraoperative photograph. Kahook Dual Blade tip visible in the anterior chamber. Gonioscope placed on the eye allows visualization of the filtration angle

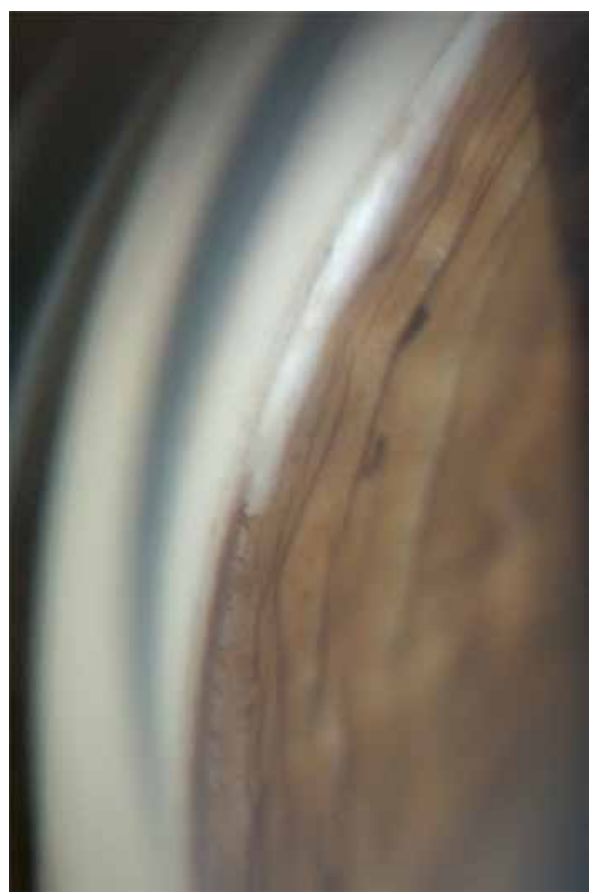

Figure 3. View of the filtration angle after the procedure. Site of incision of the trabecular meshwork visible in the upper part of the photograph; unchanged angle in the lower part

in $38 \%$ of patients. A decrease in IOP below $18 \mathrm{mmHg}$ was achieved in $58 \%$ of patients.

Changes in the number of medications used by patients

The mean number of drugs used before the procedure was $2.2 \pm 1.1$ (Table I). Six months after the surgery, it decreased to $0.88 \pm 1.0$, which corresponds to a reduction by $60 \%$ $(p<0.001)$. Figure 4 shows graphs representing changes in the analyzed parameters over time. In $82 \%$ of the eyes, the number of glaucoma medications was reduced by at least 1 . In the group of patients with IOP reduction under $20 \%$, the mean decrease in the number of medications was $1.73 \pm 0.71$.

\section{Safety profile}

On the first postoperative day, there was an increase in IOP between 25 and $34 \mathrm{mmHg}$ in a total of 11 eyes (14.6\%), 
Table I. Mean intraocular pressure (IOP) values and mean number of glaucoma medications used during 6-month follow-up

\begin{tabular}{|l|c|c|c|c|c|c|} 
& At baseline & $\begin{array}{c}1 \text { day } \\
\text { postoperatively }\end{array}$ & $\begin{array}{c}1 \text { week } \\
\text { postoperatively }\end{array}$ & $\begin{array}{c}1 \text { month } \\
\text { postoperatively }\end{array}$ & $\begin{array}{c}3 \text { months } \\
\text { postoperatively }\end{array}$ & $\begin{array}{c}6 \text { months } \\
\text { postoperatively }\end{array}$ \\
\hline Mean IOP \pm SD $(\mathrm{mmHg})$ & $21.90 \pm 3.9$ & $19.53 \pm 3.7$ & $20.38 \pm 5.7$ & $18.25 \pm 3.7$ & $17.76 \pm 2.8$ & $18.14 \pm 3.2$ \\
\hline Mean number of glaucoma medications & $2.2 \pm 1.1$ & $0.47 \pm 0.7$ & $0.8 \pm 0.9$ & $0.82 \pm 0.9$ & $0.76 \pm 0.9$ & $0.88 \pm 1.0$ \\
\hline
\end{tabular}

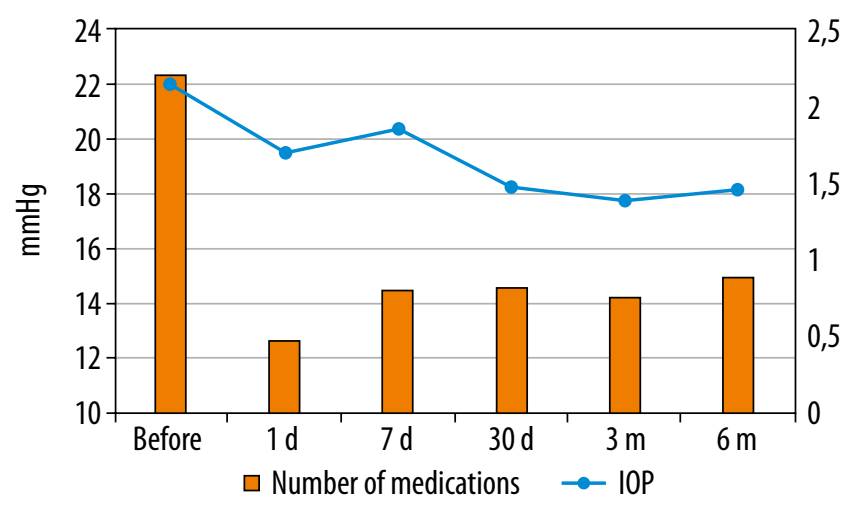

Figure 4. Changes in mean IOP values and mean number of glaucoma medications used during 6-month follow-up

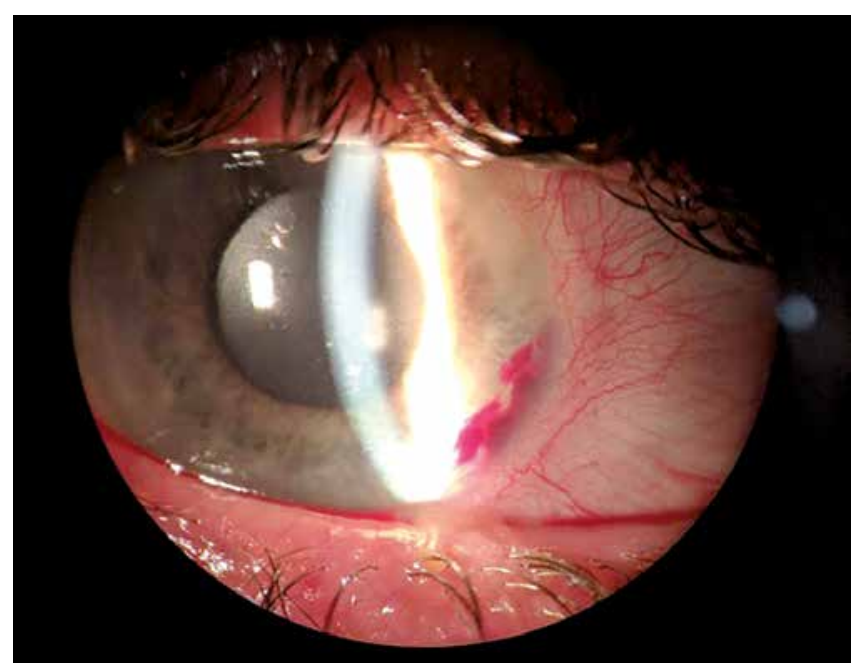

Figure 5. Blood clot in the anterior chamber in one of the patients, noted on day 1 postoperatively

and over $35 \mathrm{mmHg}$ in 12 eyes (16.0\%). All the patients experienced slight intraoperative bleeding at Schlemm's canal opening. On the first postoperative day, diffuse blood cells in the anterior chamber were observed in 5 eyes (6.6\%), and a slight blood clot was detected in $8(10.7 \%)$ patients (Figure 5$)$. In one eye $(1.3 \%)$, IOP could not be stabilized despite the introduction of pharmacotherapy, and laser cyclodestruction was additionally performed.

\section{DISCUSSION}

The effect of cataract phacoemulsification on the value of IOP has been evaluated in multiple studies. In healthy people undergoing cataract surgery, the average reduction in IOP was $1.0-4.0 \mathrm{mmHg}$ [12-14]; in individual studies the effect was slightly higher [15]. The degree of IOP reduction has been shown to be proportional to the baseline value $[13,15,16]$.
The small discrepancy between the studies can probably be attributed, among other factors, to different mean baseline IOP values. In patients with POAG, similar analyses of long-term effects showed a slightly different IOP reduction one year after the procedure: by $1.15 \pm 3 \mathrm{mmHg}(6.8 \pm 18.1 \%)$ [17], by 1.0 \pm 5.5 [12], or by $13 \%$ [18]. A large metaanalysis found that the mean reduction in IOP noted at 6, 12, 24 and 36 months after the surgery was $12 \%, 14 \%, 15 \%$ and $9 \%$ from the baseline, respectively [14]. Similarly to healthy subjects, the reduction of IOP in patients with POAG was proportionally greater in patients with higher baseline IOP. It was also found that in patients with POAG the shallower the anterior chamber and the narrower the angle of filtration, the greater the hypotensive effect of the procedure [19].

The above-mentioned metaanalysis demonstrated a reduction in the number of glaucoma medications used by patients by an average of 0.66 at 6 months after cataract phacoemulsification, and by $0.58,0.53$ and 0.38 at one, two and three years after the procedure, respectively. However, the effect of reduced use of glaucoma medications appears to diminish over time [20]. In a recently published paper, a decrease in the number of medications was achieved in $17.1 \%$ of patients. A total of $7.2 \%$ patients required additional glaucoma medications, and in $75.7 \%$ of patients with POAG the number of medications remained unchanged [17]. Reducing the dependence on glaucoma medications limits their adverse effects on the ocular surface [14], leading to improved patient comfort and potentially increasing therapeutic compliance.

In this study, the mean reduction in IOP was $17.53 \%$. In $38 \%$ of patients, the decrease in IOP during a follow-up of 6 months was over $20 \%$, while in $52 \%$ of patients it was below $18 \mathrm{mmHg}$. The relatively small reduction in IOP can be attributed to the fact that IOP in the study cohort was wellcontrolled preoperatively with eye drops. However, these values are significantly higher compared to the outcomes of cataract surgery alone, especially considering that the number of glaucoma medications used by patients was reduced by at least 1 in $82 \%$ of patients, by an average of $60 \%$. In addition, the analysis showed that in the group of patients with IOP reduction not exceeding $20 \%$, the mean decrease in the number of medications was higher than in patients with greater IOP reduction: $-1.73 \pm 0.71$ and -1.4 , respectively. Both endpoints, i.e. IOP reduction by at least $20 \%$ or less than $18 \mathrm{mmHg}$ and a decrease in the number of medications by at least one, were achieved in $24 \%$ of patients. Our findings do not differ significantly from data reported in studies published to date, evaluating the efficacy of KDB goniotomy performed in conjunction with cataract surgery [21-24]. In these studies, 
IOP decrease achieved after the KDB procedure combined with cataract phacoemulsification was $26.4-33.4 \%$ (2.1-6.93 $\mathrm{mmHg})$. The effect depended on the baseline IOP value $(17.1 \pm 4.8-20.75 \pm 8.15 \mathrm{mmHg})$. In these studies, the reduction in IOP was slightly higher than in our observation, but the decrease in the number of glaucoma medications was smaller (by an average of 0.7-1.1).

Following isolated $\mathrm{KDB}$ goniotomy, a mean decrease in IOP by 3.8-13.7 $\mathrm{mmHg}(21-46 \%)$ and a reduction in the number of glaucoma medications by $0.9-1.3$ were reported. A greater reduction in IOP and decrease in the number of medications were noted in the eyes with higher baseline IOP values [25].

The efficacy of reducing IOP shown in studies evaluating traditional trabectome surgery performed with a trabectome designed specifically to remove the trabecular meshwork and the inner wall of Schlemm's canal by electric cauterization with irrigation and aspiration was in the range of $35-48 \%$, with the size of the effect depending on baseline IOP [27-30]. The reduction in the number of medications used was maintained in the range of 0.8-1.7 [29]. KDB goniotomy is based on the same concept as trabectome surgery, but in principle the instrument allows for greater precision of excising the trabeculum, without leaving superfluous tissue that might induce inflammation or cause adhesions. For comparison, noninvasive SLT reduces IOP by more than $20 \%$ below baseline values in $66.7-75 \%$ of patients at 6 months after the surgery $[1,26,31]$. However, in a 5 -year follow-up, the effect persists in only $11.1-31 \%$ of patients, and declines over time $[1,26]$. In some of the studies, the number of glaucoma medications used by patients was maintained at a constant level throughout the entire follow-up period [31] or other medications were added to the regimen, as needed [26]. In other studies, the number of medications was reduced as far as possible (on average by -0.162 (SD 1.21) [32].

A metaanalysis of 5 studies (248 patients) evaluating the efficacy of the iStent device - a micro-bypass stent connecting the anterior chamber with Schlemm's canal - showed a 22\% reduction in IOP over an 18-month follow-up in patients with one iStent, a 30\% reduction in IOP during 6 months after the procedure in patients with two iStents, and a $41 \%$ reduction in IOP over a 6-month follow-up in patients with three iStents. The studies consistently demonstrated a greater reduction in the use of glaucoma medications in patients with more iStents (by an average of -1.68 for one implant, -1.88 for two, and -2.0 for three implants), but the exact degree of reduction varied significantly between studies and hence requires further observation [33]. However, an increase in the number of implants raises the cost of the procedure. An analysis of costs associated with the implantation of a single $[34,35]$ or even a double [36] iStent compared to laser trabeculoplasty and pharmacotherapy showed that during the first year after the surgery the latter two options were more cost-efficient. However, the iStent seems to be a more costefficient approach after 5 years, especially in patients using more expensive glaucoma medications. The cost of a single disposable KDB accounts for approx. 50\% of the iStent price. Studies comparing the efficacy of the iStent treatment and KDB goniotomy (both procedures performed in conjunction with cataract phacoemulsification) showed that the degree of IOP reduction was similar for both modalities [37] or KDB goniotomy was superior to a single iStent [38, 39].

The HYDRUS II study compared IOP following cataract surgery and after the combined procedure with the implantation of a Hydrus stent - an implant placed in Schlemm's canal to improve the outflow of aqueous humor. In the second group, IOP was reduced by at least $20 \%$ in $80 \%$ of patients, but only in $46 \%$ of patients who underwent cataract surgery alone [40]. For patients with POAG who underwent GATT (gonioscopy-assisted transluminal trabeculotomy), a $30 \pm 20 \%$ reduction in IOP and a decrease in the number of glaucoma medications by $0.9 \pm 1.3$ was achieved in a 12-month followup [41].

A study comparing Trab 360 with KDB goniotomy found no significant differences in terms of mean IOP reduction. Trab 360 - a procedure involving the insertion of a microcatheter in Schlemm's canal, followed by breaking through the anterior wall of the canal and trabecular meshwork with the microcatheter - successfully reduced IOP in $84.6 \%$ of patients. For KDB goniotomy, the corresponding figure was $81.7 \%(p=0.737)$. However, IOP $\leq 18 \mathrm{mmHg}$ was achieved in more eyes in the KDB group (80.0\% [56/70] vs. $59.3 \%$ $[16 / 27], p=0.040)$ and $\leq 15 \mathrm{mmHg}(61.4 \%$ [43/70] vs. $25.9 \%$ [7/27], $p=0.003$ ) [42]. At 12 months' follow-up, ab interno canaloplasty (ABiC (Ellex)) led to post-surgery reduction in mean IOP from $18.6 \pm 6.4 \mathrm{mmHg}$ to $14.1 \pm 3.7 \mathrm{mmHg}$ after 6 months, and $12.9 \pm 2.0 \mathrm{mmHg}$ after 12 months. The mean number of glaucoma medications was reduced from 2.0 to 1.0 [43].

For trabeculectomy with mitomycin $\mathrm{C}$, a reduction in IOP exceeding $30 \%$ was shown in up to $39.4 \%$ of patients during a 4-year follow-up [44]. The efficacy of filtering valves determined by studies was similar to trabeculectomy, but the method was associated with a greater number of glaucoma medications used by patients after the surgery, both at 6 months and 5 years after the procedure [45].

In the reported study, all patients successfully underwent combined trabectome surgery with cataract phacoemulsification. There was slight intraoperative bleeding at the site of Schlemm's canal opening in all eyes. One patient (1.3\%) required another glaucoma procedure. There were no severe complications associated with the procedure. Intraoperative bleeding, which occurred in all patients, is a natural consequence of the surgical technique applied for the procedure. Our observations do not differ from complications described in the literature after similar combined procedures [21]. The most commonly reported complications developing after KDB treatment combined with phacoemulsification include eye irritation (2.8-11.4\%), discomfort and pain (1.4-8.6\%), blurred vision (1.4-17.1\%), and postoperative IOP increases (2.8-17.1\%). Most of them resolve within 2-3 days after the procedure. Some patients developed cystic macular edema 
(1.4-5.7\%) [21, 22, 46]. Cyclodialysis was reported in one patient [47]. In other MIGS procedures increasing trabecular outflow, the most commonly reported were minor complications such as hyphema (23\%) and irritation, which resolved during the first 14 days after the procedure. Short-term increases in IOP were observed in $7.4 \%$ of patients, and macular edema in $11.1 \%$. The rate of complications does not vary significantly between the different types of procedures [21, $41,42,48]$. Peripheral focal anterior adhesions with no significance for the hypotensive effect were reported in $12 \%$ of patients treated with Hydrus implants [40].

A considerably higher rate of complications, especially of severe nature, is noted after trabeculectomy [49]. An increased risk is observed not only for intraoperative but also postoperative complications related to the filtration bleb, including infectious and metabolite-associated complications, or the development of malignant glaucoma. There is also an elevated risk of hypotony and related complications such as maculopathy, choroidal detachment or the development of cataract in phakic eyes [50]. With drainage implants there is a risk of complications related to the implant itself. Excessive filtration during the postoperative period may cause the anterior chamber to become shallow, in which case the tube may contact the endothelium. In addition, dislocations of the implant under the conjunctiva or inside the eyeball have been reported. Also, there have been cases of conjunctival perforation by the implant [4].

\section{CONCLUSIONS}

$A b$ interno goniotomy using the Kahook Dual Blade performed in conjunction with cataract surgery leads to a significant IOP reduction and a decrease in the number of glaucoma medications used. It is a safe therapeutic modality in patients with early and intermediate glaucoma who do not require intensive treatment. The procedure should be considered in patients who fail to comply with the recommendations regarding pharmacological glaucoma treatment or are allergic to glaucoma medications. A major advantage of the procedure is the fact that it does not significantly change the anatomy of the eye, and does not limit the possibility of performing other procedures in the future. The data obtained so far are promising, however, further observation of patients is necessary in order to reliably evaluate the long-term effects.

\section{DISCLOSURE}

The authors declare no conflict of interest.

\section{References}

1. Leahy KE, White AJ. Selective laser trabeculoplasty: current perspectives. Clin Ophthalmol 2015; 9: 833-841.

2. Ishida K. Update on results and complications of cyclophotocoagulation. Curr Opin Ophthalmol 2013; 24: 102-110.

3. Kumar H, Mansoori T, Wariri GB, et al. Lasers in glaucoma. Indian J Ophthalmol 2018; 66: 1539-1553.

4. Gedde SJ, Schiffman JC, Feuer WJ, et al.; Tube versus Trabeculectomy Study Group. Treatment outcomes in the Tube Versus Trabeculectomy (TVT) study after five years of follow-up. Am J Ophthalmol 2012; 153: 789-803.e2.

5. Gedde SJ, Herndon LW, Brandt JD, et al.; Tube versus Trabeculectomy Study Group. Postoperative complications in the Tube Versus Trabeculectomy (TVT) study during five years of follow-up. Am J Ophthalmol 2012; 153: 804-814.e1.

6. Richter GM, Coleman AL. Minimally invasive glaucoma surgery: current status and future prospects. Clin Ophthalmol 2016; 10: $189-206$.

7. Saheb H, Ahmed IIK. Micro-invasive glaucoma surgery: current perspectives and future directions. Curr Opin Ophthalmol 2012; 23: 96-104.

8. Brubaker RF. Measurment of uveoscleral outflow in humans. J Glaucoma 2001; 10 (5 Suppl 1): S45-48.

9. Johnson M. What controls aqueous humour outflow resistance? Exp Eye Res 2006; 82: 545-557.

10. Dorairaj SK, Kahook MY, Williamson BK, et al. A multicenter retrospective comparison of goniotomy versus trabecular bypass device implantation in glaucoma patients undergoing cataract extraction. Clin Ophthalmol 2018; 12: 791-797.

11. Seibold LK, Soohoo JR, Ammar DA, Kahook MY. Preclinical investigation of ab interno trabeculectomy using a novel dual-blade device. Am J Ophthalmol 2013; 155: 524-529.

12. Baek SU, Kwon S, Park IW, Suh W. Effect of phacoemulsification on intraocular pressure in healthy subjects and glaucoma patients. J Korean Med Sci 2019; 34: e47.

13. Mansberger SL, Gordon MO, Jampel H, et al. Reduction in intraocular pressure after cataract extraction: the Ocular Hypertension Treatment Study. Ophthalmology 2012; 119: 1826-1831.

14. Armstrong JJ, Wasiuta $T$, Kiatos $E$, et al. The effects of phacoemulsification on intraocular pressure and topical medication use in patients with glaucoma: a systematic review and meta-analysis of 3-year data. J Glaucoma 2017; 26: 511-522.

15. Poley BJ1, Lindstrom RL, Samuelson TW. Long-term effects of phacoemulsification with intraocular lens implantation in normotensive and ocular hypertensive eyes. J Cataract Refract Surg 2008; 34: 735-742.

16. BilakS, Simsek A, Capkin M, et al. Biometric and intraocular pressure change after cataract surgery. Optom Vis Sci 2015; 92: 464-470.

17. Majstruk L, Leray $B$, Bouillot $A$, et al. Long term effect of phacoemulsification on intraocular pressure in patients with medically controlled primary open-angle glaucoma. BMC Ophthalmol 2019; 19: 149.

18. Chen PP, Lin SC, Junk AK, et al. The Effect of phacoemulsification on intraocular pressure in glaucoma patients: a report by the American Academy of Ophthalmology. Ophthalmology 2015; 122: 1294-1307.

19. Lin SC, Masis M, Porco TC, Pasquale LR. Predictors of intraocular pressure after phacoemulsification in primary open-angle glaucoma eyes with wide versus narrower angles (An American Ophthalmological Society Thesis). Trans Am Ophthalmol Soc 2017; 115: T6 [published correction appears in Trans Am Ophthalmol Soc 2018; 115: T6C1].

20. Noecker RJ, Herrygers LA, Anwaruddin R. Corneal and conjunctival changes caused by commonly used glaucoma medications. Cornea 2004; 23: 490-496.

21. Barry M, Alahmadi WM, Alahmadi M, et al. The safety of the Kahook Dual Blade in the surgical treatment of glaucoma. Cureus 2020 12: e6682. 
22. Greenwood MD, Seibold LK, Radcliffe NM, et al. Goniotomy with a single-use dual blade: short-term results. J Cataract Refract Surg 2017; 43: 1197-1201.

23. Hirabayashi MT, King JT, Lee D, et al. Outcome of phacoemulsification combined with excisional goniotomy using the Kahook Dual Blade in severe glaucoma patients at 6 months. Clin Ophthalmol 2019; 13: 715-721.

24. Dorairaj SK, Seibold LK, Radcliffe NM, et al. 12-Month outcomes of goniotomy performed using the Kahook Dual Blade combined with cataract surgery in eyes with medically treated glaucoma. Adv Ther 2018; 35: 1460-1469.

25. Berdahl JP, Gallardo MJ, ElMallah MK, et al. Six-month outcomes of goniotomy performed with the Kahook Dual Blade as a stand-alone glaucoma procedure. Adv Ther 2018; 35: 2093-2102.

26. Woo DM, Healey PR, Graham SL, Goldberg I. Intraocular pressure-lowering medications and long-term outcomes of selective laser trabeculoplasty. Clin Experiment Ophthalmol 2015; 43: 320-327.

27. Minckler DS, Baerveldt G, Alfaro MR, Francis BA. Clinical results with the trabectome for treatment of open-angle glaucoma. Ophthalmology 2005; 112: 962-967.

28. Vold SD. Impact of preoperative intraocular pressure on trabectome outcomes: a prospective, non-randomized, observational, comparative cohort outcome study. Clin Surg Ophthalmol 2010; 28: 11.

29. Mizoguchi T, Nishigaki S, Sato T, et al. Clinical results of trabectome surgery for open-angle glaucoma. Clin Ophthalmol 2015; 9: 1889-1894.

30. Shoji N, Kasahara M, lijima A, et al. Short-term evaluation of trabectome surgery performed on Japanese patients with open-angle glaucoma. Jpn J Ophthalmol 2016; 60: 156-165.

31. Martinez-de-la-Casa JM, Garcia-Feijoo J, Castillo A, et al. Selective vs argon laser trabeculoplasty: hypotensive efficacy, anterior chamber inflammation, and postoperative pain. Eye (Lond) 2004; 18: 498-502.

32. Kent SS, Hutnik CML, Birt CM, et al. A randomized clinical trial of selective laser trabeculoplasty versus argon laser trabeculoplasty in patients with pseudoexfoliation. J Glaucoma 2015; 24:344-347.

33. Malvankar-Mehta MS, Chen YN, lordanous Y, et al. iStent as a solo procedure for glaucoma patients: a systematic review and meta-analysis. PLoS One 2015; 10: e0128146.

34. Ordóñez JE, Ordóñez A, Osorio UM. Cost-effectiveness analysis of iStent trabecular micro-bypass stent for patients with open-angle glaucoma in Colombia. Curr Med Res Opin 2019; 35: 329-340.

35. Ngan K, Fraser E, Buller S, Buller A. A cost minimisation analysis comparing iStent accompanying cataract surgery and selective laser trabeculoplasty versus topical glaucoma medications in a public healthcare setting in New Zealand. Graefes Arch Clin Exp Ophthalmol 2018; 256:2181-2189.

36. Berdahl JP, Khatana AK, Katz $\mathrm{U}$, et al. Cost-comparison of two trabecular micro-bypass stents versus selective laser trabeculoplasty or medications only for intraocular pressure control for patients with open-angle glaucoma. J Med Econ 2017; 20: 760-766.

37. Lee D, King J, Thomsen S, et al. Comparison of surgical outcomes between excisional goniotomy using the Kahook Dual Blade and iStent trabecular micro-bypass stent in combination with phacoemulsification. Clin Ophthalmol 2019; 13: 2097-2102.

38. Dorairaj SK, Kahook MY, Williamson BK, et al. A multicenter retrospective comparison of goniotomy versus trabecular bypass device implantation in glaucoma patients undergoing cataract extraction. Clin Ophthalmol 2018; 12: 791-797.

39. EIMallah MK, Seibold LK, Kahook MY, et al. 12-Month retrospective comparison of Kahook Dual Blade excisional goniotomy with istent trabecular bypass device implantation in glaucomatous eyes at the time of cataract surgery. Adv Ther 2019; 36: 2515-2527.

40. Pfeiffer N, Garcia-Feijoo J, Martinez-dela-Casa JM, et al. A randomized trial of a Schlemm's canal microstent with phacoemulsification for reducing intraocular pressure in open-angle glaucoma. Ophthalmology 2015; 122: 1283-1293.

41. Grover DS, Godfrey DG, Smith 0, et al. Gonioscopy-assisted transluminal trabeculotomy, ab interno trabeculotomy: technique report and preliminary results. Ophthalmology 2014; 121: 855-861.

42. Hirabayashi MT, Lee D, King JT, et al. Comparison of surgical outcomes of $360^{\circ}$ circumferential trabeculotomy versus sectoral excisional goniotomy with the Kahook Dual Blade at 6 months. Clin Ophthalmol 2019; 13: 2017-2024.

43. Guttman Krader C. ABiC targets all sites of outflow resistance. Ophthalmol Times 2016; 12: 35-36.

44. Jongsareejit B, Tomidokoro A, Mimura T, et al. Efficacy and complications after trabeculectomy with mitomycin C in normal tension glaucoma. Jpn J Ophthalmol 2005; 49: 223-227.

45. Tseng VL, Coleman AL, Chang MY, Caprioli J. Aqueous shunts for glaucoma. Cochrane Database Syst Rev 2017; 2017: CD004918.

46. Arnljots TS, Economou MA. Reversible cystoid macular edema following uneventful microinvasive Kahook Dual Blade goniotomy in a pseudophakic patient: a case report. J Glaucoma 2018; 27: e128-e130.

47. Shue A, Levine RM, Gallousis GM, et al. Cyclodialysis cleft associated with Kahook Dual Blade goniotomy. J Curr Glaucoma Pract 2019; 13: 74-76.

48. Buchacra 0, Duch S, Milla E, Stirbu 0. One-year analysis of the istent trabecular microbypass in secondary glaucoma. Clin Ophthalmol 2011; 5: 321-326.

49. Jampel HD, Musch DC, Gillespie BW, et al. Perioperative complications of trabeculectomy in the Collaborative Initial Glaucoma Treatment Study (CIGTS). Am J Ophthalmol 2005; 140: 16-22.

50. Yuasa $Y$, Sugimoto $Y$, Hirooka $K$, et al. Effectiveness of trabeculectomy with mitomycin ( for glaucomatous eyes with low intraocular pressure on treatment eye drops. Acta Ophthalmol 2020; 98: e81-e87. 